\title{
Inflammasome as a Therapeutic Target for Cancer Prevention and Treatment
}

\author{
Huyen Trang Ha Thi, Suntaek Hong \\ Department of Biochemistry, Gachon University College of Medicine, Incheon, Korea
}

\begin{abstract}
Chronic inflammation is a critical modulator of carcinogenesis through secretion of inflammatory cytokines, which leads to the formation of an inflammatory microenvironment. In this process, the inflammasome plays an important role in the expression and activation of interleukin (IL)-1 $\beta$ and IL-18 to promote cancer development. The inflammasome is a multiprotein complex consisting of several nucleotide-binding domain and leucine-rich repeat containing receptor, adaptor proteins, and caspase 1 (CASP1). It senses the various intracellular (damage-associated molecular patterns) and extracellular (pathogen-associated molecular patterns) stimuli. A primed inflammasome recruits adaptor proteins, activates CASP1 to enhance the proteolytic cleavage of pro-IL-1 $\beta$ and IL-18, and sends the signal to respond to each insult. Depending on stimuli and cell contexts, several inflammasomes are closely associated with the initiation and promotion of carcinogenesis. In contrast, inflammasomes also show an ambivalent effect on carcinogenesis by enhancing inflammatory cell death (pyroptosis) and repairing damaged tissues. Although the inflammasome plays a controversial role in carcinogenesis, it may be a promising target for human cancer prevention and treatment. A more in-depth study on the role of the inflammasome in carcinogenesis, based on stimuli, cell contexts, and cancer stages, can lead to the development of novel therapeutic strategies against malignant human cancers.
\end{abstract}

(J Cancer Prev 2017;22:62-73)

Key Words: Inflammasomes, Cancer, NLR proteins, Therapeutics

\section{INTRODUCTION}

Inflammation is a host defense system to protect the body against either viral, bacterial, and fungal infections or internal insults. ${ }^{1}$ This process should be tightly regulated to keep the homeostasis of the host's immune system. When acute inflammation is maintained for a very short time, it eliminates and repairs damaged cells and injured tissues through the recruitment of immune cells and the expression of inflammatory mediators. When maintained for longer periods, inflammation becomes chronic and creates problems in the human body. Chronic inflammation is a cause of many diseases, including diabetes, arthritis, inflammatory bowel disease, autoimmune diseases, and cancer. An inflammatory microenvironment is considered a hallmark of carcinogenesis. ${ }^{2}$ The involvement of immune cells in tumor formation was suggested after finding leukocyte infiltration in breast cancer samples already in the 19th century. Many researchers reported that chronic inflammation promotes carcinogenesis through resistance to growth inhibition, formation of blood vessel, escape from apoptosis, and initiation of metastasis. ${ }^{3.4}$ About $20 \%$ of human cancers are closely associated with chronic inflammation. ${ }^{4}$

In the early stage of carcinogenesis, infiltrated immune cells release reactive oxidative molecules to enhance the epigenetic mutation in oncogenes and tumor suppressor genes. Inflammation-induced oxidative stress also induces inactivation of the mismatch repair system, leading to the insertion of mutation into important genes. ${ }^{5}$ Another mechanism for the promotion of

Received May 17, 2017, Revised May 27, 2017, Accepted May 29, 2017

Correspondence to: Suntaek Hong

Department of Biochemistry, Gachon University College of Medicine, 155 Gaetbeol-ro, Yeonsu-gu, Incheon 21999, Korea

Tel: +82-32-899-6311, Fax: +82-32-899-6039, E-mail: sthong@gachon.ac.kr, ORCID: Suntaek Hong, http://orcid.org/0000-0001-9338-5971

Copyright (C) 2017 Korean Society of Cancer Prevention

(c) This is an Open Access article distributed under the terms of the Creative Commons Attribution Non-Commercial License (http://creativecommons.org/licenses/by-nc/4.0) which permits unrestricted non-commercial use, distribution, and reproduction in any medium, provided the original work is properly cited. 
cancer initiation by inflammation is the stimulation of stem cell-like phenotypes. ${ }^{6}$ In late stage carcinogenesis, cytokines and chemokines are secreted from the inflammatory environment to support cell survival and invasiveness. In particular, TGF- $\beta$ is a key cytokine to enhance the epithelial-mesenchymal transition and to promote the metastasis of cancer cells. ${ }^{7}$ Metastasis of cancer cells into specific tissues or organs is also tightly regulated by the expression of chemokines. ${ }^{8}$ For example, breast cancer cells expressing CXCR4 mainly migrate to the lung, bone, and lymph node, which contain high levels of CXCL12. ${ }^{9}$ Upregulation of CXCR3 in colorectal cancer increases the metastasis into preferred sites, including liver, lung, and lymph nodes, which release high amounts of CXCL10. ${ }^{10}$ To identify the molecular mechanism of the relationship between inflammation and carcinogenesis, we need to understand the role of the key components in the inflammation signaling pathway.

\section{INFLAMMASOME SIGNALING PATHWAY}

When the human body is exposed to microbes or exogenous stimuli, pattern recognition receptors (PRRs) play a protective role as gatekeepers in the defense system. The PRR family consists of various members, including Toll-like receptors (TLRs), nucleotide-binding domain and leucine-rich repeat containing receptors (NLRs), retinoic acid-inducible gene I-like RNA helicases, C-type lectins, and absent in melanoma (AIMs) like receptors. ${ }^{11,12}$ In a first step, PRRs recognize the debris of invaders, including pathogen-associated molecular patterns (PAMPs) and damage-associated molecular patterns (DAMPs), to initiate the innate and adaptive immunity. Of the numerous PRR members, we focus on the role of NLRs in the inflammatory signaling pathway and carcinogenesis due to their ability to form the multiprotein complex inflammasome. ${ }^{13}$

The NLR families consist of 22 large members in the human body. ${ }^{14}$ The NLR proteins consist of 3 major domains: a N-terminal protein-protein interaction domain, a middle nucleotide-binding oligomerization (NACHT) domain, and a C-terminal leucine-rich repeat (LRR) domain. The N-terminal domain is responsible for the recruitment of downstream signaling adaptors with a Pyrin domain, a caspase recruitment and activation domain (CARD), or a baculovirus inhibitor of apoptosis repeat domain. ${ }^{15}$ The NACHT domain involves the recruitment of pro-caspase 1 (CASP1) through interaction with an adaptor protein, an apoptosis-associated speck-like protein containing a CARD domain (ASC). The central domain also regulates the self-oligomerization of NLRs. The LRR domain determines the specificity of ligands, depending on the types of NLRs. After interaction with specific PAMP or DAMP, NLRs are oligomerized to homo- or hetero-complexes to recruit the pro-CASP1 via the ASC adaptor protein. Pro-CASP1 is subsequently activated through self-cleavage to form a mature p10-p20 complex. Active CASP1 regulates the generation of mature interleukin (IL)-1 $\beta$ and IL-18 from inactive forms to induce the inflammatory form of cell death (pyroptosis) or to recruit other immune cells. ${ }^{16}$ Matured IL-1 $\beta$ and IL-18 are important promoters of various inflammatory signaling through NF- $\mathrm{\kappa B}$ and mitogen-activated protein kinase (MAPK) activation. Secreted cytokines from immune cells also generate the inflammatory microenvironment in infiltrated tissues. This is called the canonical inflammasome pathway involving NLR, ASC, and CASP1. In contrast to the canonical pathway, the non-canonical pathway works with CASP8 or CASP11. Upon stimulation with fungi or mycobacteria, the dectin-1 receptor activates CASP8 to regulate the activation of IL-1 $\beta .{ }^{17}$ An involvement of CASP8 was also found in lipopolysaccharide-stimulated macrophages or dendritic cells and apoptosis with inflammasome complex. ${ }^{18}$ An infection with enteric bacteria, such as Escherichia coli, Vibrio cholera, or Citrobacter rodentium, induces the pyroptotic cell death through the proteolytic cleavage and activation of CASP11. ${ }^{19,20}$ It is not fully understood yet what causes this difference in the recruitment of the caspases upon various stimuli.

The inflammasome was first identified as a multiprotein complex containing CASP1, CASP5, Pycard/Asc, and NLR protein 1 (NLRP1) and named by Tschopp's group through immunoprecipitation experiments. ${ }^{13}$ Since then, many inflammasomes were isolated and showed the differences in ligands, components, and roles in the immune system. They are classified by the presence of NLR in the complex, for example, NLRP1, NLRP3, NLR family CARD domain containing 3 (NLRC3), NLRC4, NLR family apoptosis inhibitory protein (NAIP), and NLRP6. An important inflammasome of the pyrin and HIN domain family member is AIM2. ${ }^{21,22}$ The function of each inflammasome is different and depends on ligands and receptors. We summarize the types of inflammasomes, ligands, and functions in Table 1.

NLRP1 inflammasome is a sensor for muramyl dipeptide and Bacillus anthracis lethal toxin and forms a complex with ASC and CASP1. ${ }^{23,24}$ The abnormal regulation of NLRP1 is associated with many human diseases. For example, NLRP1-mediated pyroptosis is detected in cortical neurons of Alzheimer's disease model. NLRP1 also activates CASP1, which results in the acute inflammation in lungs and has catastrophic consequences for the organism. ${ }^{25,26}$ Our understanding of the inflammasome stems 
Table 1. Types and activities of components forming inflammasome

\begin{tabular}{|c|c|c|c|}
\hline Sensor & Adaptor & Function & Ligand \\
\hline NLRP1 & ASC, CASP 1 & $\begin{array}{l}\text { - Recruits CASP1 through CARD domain } \\
\text { - Induces maturation of inactive cytokines } \\
\text { - Induces pyroptotic cell death }\end{array}$ & $\begin{array}{l}\text { Lethal toxin, Muramyl dipeptide, } \\
\text { ATP }\end{array}$ \\
\hline NLRP3 & ASC, CASP1 & $\begin{array}{l}\text { - Forms complex with ASC and CASP1 } \\
\text { - Is activated by released cathepsin B from damaged lysosomes } \\
\text { - Senses ROS directly or indirectly to activate the downstream signaling } \\
\text { - Involved in } \mathrm{Th}_{2} \text { and } \mathrm{Th}_{17} \text { polarization }\end{array}$ & $\begin{array}{l}\text { Bacterial/viral DNA, extracellular } \\
\text { ATP, monosodium urate, } \\
\text { amyloid- } \beta \text {, silica, asbestos }\end{array}$ \\
\hline NLRC4 & $\begin{array}{l}\text { ASC, CASP1, } \\
\text { NAIP }\end{array}$ & $\begin{array}{l}\text { - Induces CASP1 proteolysis and cell death in macrophages } \\
\text { - Is highly expressed in hematopoietic tissues and cells } \\
\text { - Discriminates commensal bacteria from pathogens }\end{array}$ & $\begin{array}{l}\text { Flagellin and type III secretion } \\
\text { system of bacteria }\end{array}$ \\
\hline NAIP & NLRC4 & $\begin{array}{l}\text { - Forms complex with NLRC4 } \\
\text { - Involves in bacterial sensing and pyroptosis in macrophages and } \\
\text { epithelial cells } \\
\text { - Protects neurons from calcium-induced cell death via CASP3-dependent } \\
\text { manner }\end{array}$ & $\begin{array}{l}\text { Cprl T3SS needle protein, } \\
\text { Flagellin of Legionella }\end{array}$ \\
\hline NLRP6 & ASC, CASP1 & $\begin{array}{l}\text { - Regulates intestinal homeostasis by sensing alteration in gut flora } \\
\text { - Recruits ASC to activate CASP } 1 \text { and release IL-1 } \beta \text { and IL-18 }\end{array}$ & Not identified \\
\hline AIM2 & ASC, CASP 1 & $\begin{array}{l}\text { - Recognizes cytoplasmic double stranded DNA to activate inflammasome } \\
\text { pathway } \\
\text { - Forms ASC pyroptosome to induce cell death through activation of } \\
\text { CASP1 and IL-1 } \beta\end{array}$ & $\begin{array}{l}\text { Bacterial/viral DNA, self DNA of } \\
\text { apoptotic cell }\end{array}$ \\
\hline
\end{tabular}

NLRP, nucleotide-binding domain and leucine-rich repeat containing receptor protein; NLRC, nucleotide-binding domain and leucine-rich repeat containing receptor family CARD domain containing; NAIP, nucleotide-binding domain and leucine-rich repeat containing receptor family apoptosis inhibitory protein; AIM, absent in melanoma; ASC, apoptosis-associated speck-like protein containing a caspase recruitment and activation domain; CASP1, caspase 1; CARD, caspase recruitment and activation domain; ROS, reactive oxygen species; IL, interleukin.

from the study of the NLRP3 inflammasome. NLRP3 recognizes various stimuli, including bacteria and their debris, and endogenous damage signals. NLRP3 can sense invaders, such as Sendai virus, Influenza virus, Candida albicans, Staphylococcus aureus, and bacterial pore-forming toxins. ${ }^{27-29}$ NLRP3 also recognizes extracellular ATP, monosodium urate, and hyaluronan to activate the inflammatory signaling. ${ }^{28,30}$ To deliver a common signal from diverse inputs, NLRP3 uses a combined mechanism, including reactive oxygen species (ROS), mitochondrial dysfunction, and potassium and calcium channel fluxes. ${ }^{31,32}$ Mitochondria-derived ROS can activate the NLRP3 inflammasome by de-ubiquitination of NLRP3 through TLR4/MyD88 signaling. ${ }^{33}$ This paper also suggests that extracellular ATP can prime the NLRP3 inflammasome through deubiquitination. A recent paper described that NLRP3 inflammasome regulates cholestasis-associated sepsis via sensing of bile acid and ATP. ${ }^{34}$ NLRC4 can discriminate between commensal bacteria and pathogens to suppress their colonization and to coordinate intestinal epithelial cell expulsion with lipid mediator and cytokine secretion. ${ }^{35-37}$ Unlike other NLRs, NLRP6 has not been confirmed yet as true inflammasome. Although NLRP6 deficient mice show a resistance to infection with bacterial pathogens and to the defective autophagy in cells, binding ligands and detailed upstream signaling are not identified yet. ${ }^{38}$ NLRP6 is highly expressed in intestinal cells to regulate the colonic microbial ecology and functions as negative regulator of NF-KB and MAPK signaling. AIM2 was originally isolated as a tumor suppressor gene in melanoma and later validated as non-NLR inflammasome. $^{39}$ The HIN-200 domain of AIM2 senses double-strand DNA of bacterial origin and self-DNA of dead cells to induce the apoptotic or pyroptotic cell death. ${ }^{18,40}$ The specific role of the AIM2 inflammasome in brain injuries was confirmed with AIM2 knockout mice after bacterial infection. ${ }^{41}$ Although many reports have been published about the role of inflammasomes in inflammatory signaling and human diseases, their signaling pathways from ligands, receptors, and adaptors to effectors are not fully understood.

\section{DUAL ROLE OF INFLAMMASOME IN CANCER}

Tumor cells are equipped with the ability to escape from or are resistant to programmed cell death mediated by apoptosis ${ }^{42}$ and necrosis. ${ }^{43}$ Tumor cells are also ignited by other lethal cascades to get into an immortal phase. 'Pyroptosis', an inflammasome-dependent cell death is started by many pathological stimuli, such as stroke, heart attack, or cancer, and is critical for regulating 
microbial infections. ${ }^{44}$ It is reasonable to assume that pyroptosis might contribute to cell-autonomous tumor suppression and is suppressed in carcinogenesis. ${ }^{16}$ CASP1, a key player in pyroptosis, was shown to be a tumor suppressor in a variety of human cancers. A significant decrease of CASP1 was observed in epithelial cells from prostate cancer tissues. ${ }^{45}$ Conversely, overexpression of CASP1 in prostate cancer cell enhances the sensitivity to irradiation-induced cell death in vitro. ${ }^{46} \mathrm{CASP} 1$ was also downregulated in ovarian cancer, demonstrating the downregulation of this caspase protein as a novel mechanism to enhance the resistance of cancer cell to apoptosis. ${ }^{47}$ Most findings on the role of inflammasomes in cancer are based on the application of inflammasome-deficient mice as chemical-induced carcinogenesis models. Roles of the inflammasome in colorectal cancer were defined by using a colitis-associated colon cancer (CAC) in the azoxymethane (AOM) and dextran-sulphate sodium (DSS) model. CASP1 deficient and NLRC4 deficient mice developed a large number of tumors and a more aggressive phenotype with the invasion of tumor cells compared to wild-type mice in the AOM-DSS colon cancer model. Both $\mathrm{CASP}^{-/-}$and $\mathrm{NLRC}^{-/-}$mice showed significantly enhanced proliferation in the early phase of the inflammation-induced carcinogenesis and reduced cell death in cancers instead of inflammation modification. ${ }^{48}$ These findings support the central role of CASP1 in the prevention of colonic inflammation-induced tumor formation by mediating of the NLRC4 inflammasome. The inflammasome was also involved in UV radiation B (UVB) irradiation-induced inflammation by the constitutive expression of pro-IL-1 $\beta$ and IL-18 in keratinocytes. ${ }^{49}$ CASP1 activity is required for UVB-induced apoptosis in human keratinocyte, which further supports this finding. ${ }^{50}$ This study also indicates an early activation of inflammasome followed by apoptosis in UVB-irradiated human primary keratinocytes. Therefore, CASP1-mediated apoptosis may contribute to remove the premalignant cells and block the carcinogenesis process.

NLR family members participate in innate immune signaling through activation or inhibition of the inflammasome. Dysregulation of NLR leads to various inflammatory diseases and auto-inflammatory immune disorders. ${ }^{51}$ NLR plays dual roles as a tumor suppressor or tumor promoter in the initiation, progression, and regression of cancer. NLRP3 is the best studied member of the NLR family. NLRP3 and CASP1 deficient mice were first found to be highly susceptible to AOM/DSS-induced colorectal tumor formation. ${ }^{52}$ The work suggested that $\mathrm{NLRP3}^{-1-}$ and $\mathrm{CASP}^{-/-}$mice, but not NLRC4 ${ }^{-/-}$, showed an increase in colitis and CAC with a decrease in IL-18 expression during inflammatory processes. Moreover, expression of NLRP3 in hematopoietic cells, not epithelial cells or stromal cells, is crucial for protection against increased carcinogenesis. These data suggest that the NLRP3 inflammasome acts as a negative regulator in chemical-induced colorectal carcinogenesis. Another group found that the deficiency of NLRP3 or CASP1 in mice results in the loss of epithelial integrity by increasing the leukocyte infiltration and chemokine production in the colon, leading to higher mortality rates. Thus, the NLRP3 inflammasome is important for the maintenance of intestinal homeostasis and the protection against inflammatory diseases. ${ }^{53}$ The NLRP3 inflammasome-mediated IL-18 production is required for the maturation of hepatic natural killer (NK) cells to enhance immunosurveillance against metastasis of colonic tumor cells into the mouse liver. ${ }^{54}$ In addition, IL-18 can suppress the intestinal carcinogenesis at late stages by inhibiting the expression of soluble IL-22 receptor and IL-22 binding proteins. ${ }^{55}$ The NLRP3 inflammasome also exerts tumor suppressive functions with an adaptive immune response. ATP from the dying tumor cells could bind to purinergic receptor P2X, ligand-gated ion channel, 7 (P2RX7) receptors on dendritic cells to activate NLRP3 inflammasome and IL-1 $\beta$ receptor signaling. The lack of NLRP3 in mice results in the loss of IL-1 $\beta$ production and suppresses the effective $\mathrm{CD} 8{ }^{+}$T-cell response against tumors. ${ }^{56}$ Anti-cancer effects of NLRP3 were also reported for the chemical-induced skin carcinogenesis model. Chow and colleagues showed that wild-type mice have increased cancer formation rates than NLRP3 $3^{-/-}$mice, demonstrating that NLRP3 is an important suppressor of NK cell-mediated regulation of carcinogenesis and metastases. ${ }^{57}$ Wei et al. ${ }^{58}$ also demonstrated either completely lost or markedly downregulation of NLRP3 in human hepatocellular carcinoma (HCC). Loss of NLRP3 highly correlated with poor HCC pathological condition and advanced stage of cancer. These findings support the idea that the NLRP3 inflammasome functions as tumor suppressor in multi-stage carcinogenesis and deregulation of the inflammasome is important for HCC progression.

The suppressive activity of other NLRs in carcinogenesis was also reported. Together with CASP1, NLRC4 was shown as a negative regulator in the CAC model. NLRC4 ${ }^{-1-}$ mice showed significantly enhanced tumor numbers and burden in comparison with wild-type mice in the chemical-induced colorectal carcinogenesis model. ${ }^{48}$ CASP1 and NLRC4 were also found to suppress tumorigenesis in the AOM/DSS model. Interestingly, NLRC4 reduced the colonic epithelial and tumor cell proliferation and enhanced the cell apoptosis, but did not affect the 
inflammation-induced AOM/DSS in mice. These data suggest that intrinsic effects in the colonic epithelial cells are the cause for the tumor promotion in $\mathrm{NLRC4}^{-/-}$mice. ${ }^{59}$ Anti-cancer roles of NLRC4 were also studied in melanoma growth using knockout mice. The subcutaneous implantation of mouse melanoma B16-F10 cells into NLRC4 ${ }^{-/-}$mice enhanced the tumor sizes and tumor number compared with controls. Similar to NLRC4, the contribution of NLRP6 to colon cancer was also investigated using a mouse model. NLRP6 ${ }^{-1-}$ mice were hypersusceptible to AOM/DSS-induced colorectal carcinogenesis compare with wild type controls. The tumor development in NLRP6-deficient mice correlated with increased intestinal epithelial proliferation, as well as inflammatory cytokine productions that are associated with increased tumorigenicity. ${ }^{60}$ These data suggested a unique role of NLRP6 in the regulation of intestinal inflammation and colon carcinogenesis. In another study, NLRP6 was shown to suppress the inflammation-associated colon carcinogenesis by regulating tissue repair. These data demonstrate that NLRP6 may potentially act as a tumor suppressor in colon carcinogenesis through regulation and maintenance of intestinal inflammation. ${ }^{61}$ Another member of the NLR family that displays a protective role in colorectal carcinogenesis is NLRP12. In the AOM/DSS-induced CAC model, wild-type mice exhibited a decreased tumor development, loss of tissue damage, and pro-inflammatory cytokine production in comparison with NLRP $12^{-/-}$mice. The regulatory role of NLRP12 has been demonstrated through regulation of NF- $\mathrm{KB}$ signaling, leading to inflammatory modification against tumorigenesis. ${ }^{62}$ In a recent study, NLRP12 was found to lower the expression in human ulcerative colitis. Lack of NLRP12 in mice caused an increased basal colonic inflammation, resulting in a less diverse microbiome, loss of protective gut commensal strains, and a greater abundance of colitogenic strains. ${ }^{63}$ Overall, chemical-induced carcinogenesis experiments demonstrate that inflammasomes have protective roles in various cancers. These tumor suppressive activities are likely dependent on inflammasome-mediated modulation of inflammatory signaling, engaging T-cell activities or cell death pathways (Fig. 1).

In contrast to the protective roles of inflammasomes in carcinogenesis, activation of inflammasome-mediated inflammatory responses also promote the carcinogenesis through suppression of the antitumor immunity in some cases. Apart from anti-cancer effects, pro-tumorigenic activities of NLRs were also markedly reported (Fig. 2). NLRP3 polymorphism has been shown to correlate with melanoma susceptibility, colorectal cancer, and myelomas survival. ${ }^{64}$ NLRP3 positively regulates the lung carcinogenesis and metastasis in the chemical-induced cancer model. In this case, NLRP3 ${ }^{-/-}$mice suppress the

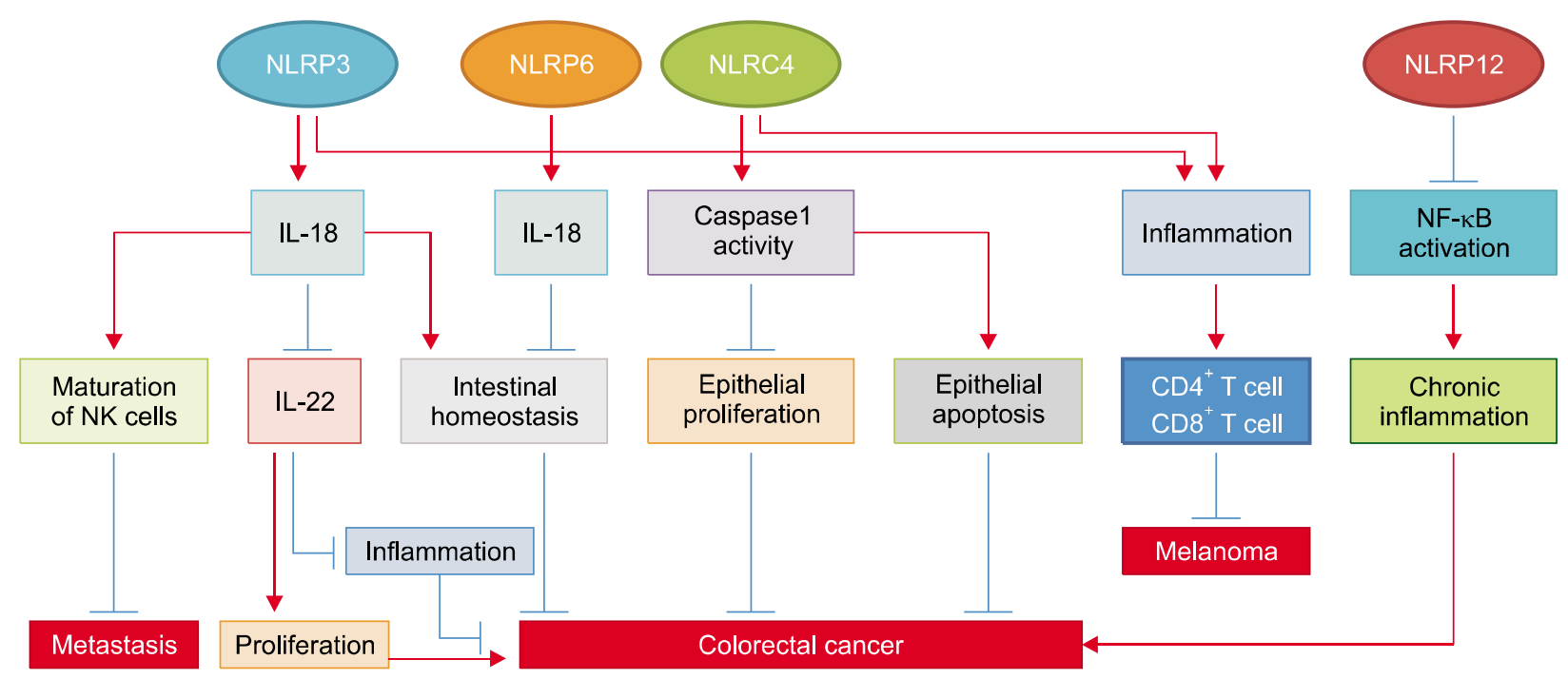

Figure 1. Protective roles of the inflammasome in cancer. Nucleotide-binding domain and leucine-rich repeat containing receptor Family Pyrin Domain Containing 3 (NLRP3) and NLRP6 regulate the induction of interleukin (IL)-18, supporting a protective role of inflammasome against colorectal cancer. IL-18 suppresses the expression of IL-22 and inhibits intestinal carcinogenesis at late stages. The NLRP3 inflammasome-mediated IL-18 production is required for the maturation of hepatic natural killer (NK) cells to enhance immunosurveillance against metastasis of colonic tumor cells into the liver. NLRP6-IL-18 axis is also involved in the maintenance of intestinal homeostasis and protection against colitis. Nucleotide-binding domain and leucine-rich repeat containing receptor family CARD domain containing 4 (NLRC4) suppresses colorectal cancer and melanoma through the enhancement of epithelial cell apoptosis and $\mathrm{T}$ cell activation. NLRP12 plays a role as tumor suppressor through the regulation of NF-KB signaling, leading to the modulation of inflammatory signaling. 


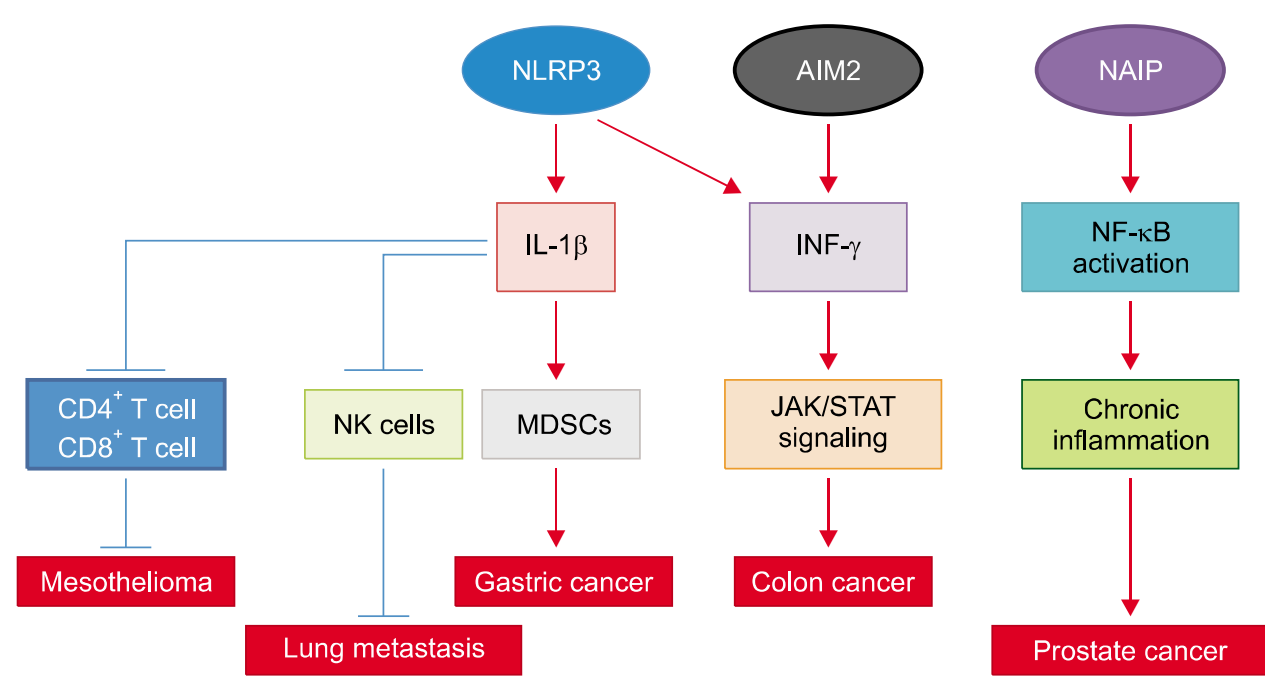

Figure 2. Pro-tumorigenic roles of the inflammasome in cancer. The nucleotide-binding domain and leucine-rich repeat containing receptor Family Pyrin Domain Containing 3 (NLRP3)-mediated interleukin (IL)-1 $\beta$ signaling suppresses the tumoricidal activity of natural killer (NK) and $\mathrm{T}$ cells to promote lung metastasis and mesothelioma. NLRP3-IL-1 $\beta$ axis results in the migration of myeloid-derived suppressor cells (MDSCs) to tumorigenic sites and promotes the gastric cancer. Overexpression of interferon (INF)- $\gamma$ by absent in melanoma 2 (AIM2) and NLRP3 also enhances Janus kinase (JAK)/STAT signaling to promote the colon cancer. NF-KB signaling activated by the nucleotide-binding domain and leucine-rich repeat containing receptor family apoptosis inhibitory protein (NAIP) inflammasome is involved in the promotion of prostate cancer through the induction of chronic inflammation.

spontaneous and experimental lung tumor metastasis compared with wild-type mice through regulation of NK cells and interferon (INF)- $\gamma$ signaling. Mechanically, the expression of NLRP 3 reduces the accumulation of CCL 5 and CXCL9 in cancer and promotes the anti-metastatic function of NK cells. Consistent with this data, no anti-metastatic effects were observed in $\mathrm{CASP}^{-/}{ }^{--}$and $\mathrm{CASP}_{1} 1^{-/-}$ mice. ${ }^{57}$ This data indicated a critical role of NLRP3 in development and metastasis of lung cancer. The pro-carcinogenic role of NLRP3 was also identified with antitumor vaccines against the poorly immunogenic melanoma cell line B16-F10. ${ }^{65}$ In this study, vaccinated NLRP3 ${ }^{-1-}$ mice showed higher survival rates compared with control mice. The survival advantage of the $\mathrm{NLRP}^{-1-}$ mouse has been explained by reduced numbers of myeloid-derived suppressor cells (MDSCs), but not through differences in dendritic cells. Knockdown of NLRP3 in MDSCs results in the suppressed migration of MDSCs to the tumor site. These data suggested a critical role of NLRP3 for the expansion of MDSCs, the migration of MDSCs to tumors, and the suppression of antitumor $\mathrm{T}$ cell activity after immune cell vaccination. ${ }^{65}$ The pro-tumor role of NLRP3 in mesothelioma was also identified. $\mathrm{NLRP3}^{-1-}$ mice and wild-type mice were applied to a model of mesothelioma by injecting asbestos fibers in solution into the peritoneum. The survival and tumor incidence were not different between mouse strains, but the infiltration of pro-inflammatory cells, including $\mathrm{NK}$ cells, $\mathrm{CD}^{+} \mathrm{T}$ cells, $\mathrm{CD}^{+} \mathrm{T}$ cells, macrophages, and neutrophils, was increased in wild-type mice compared with $\mathrm{NLRP3}^{-/-}$mice, suggesting a defective inflammatory response in NLRP3-deficient mice. NLRP3 ${ }^{-1-}$ mice suppressed the tumor growth in a two-stage carcinogen-induced skin cancer model compared with controls. These results suggest cancer promoting activity of NLRP3 in skin cancer. ${ }^{66}$ The reduction of IL-18 expression in NLRP3 or NLRP6 deficient mice indicated the pro-tumorigenic activity of NLRP3 in colon carcinogenesis. ${ }^{52.53,60}$ Additionally, treatment of recombinant IL-18 can rescue the colitis-induced injuries and suppress the colitis-associated carcinogenesis in $\mathrm{NLRP3}^{-1-}$ mice. $^{67}$ The biphasic effects of IL-18 were also published by another group. The downregulation of IL-18 is always correlated with the increase of other pro-inflammatory cytokines and chemokines, including IL-1 $\beta$, IL- 6 , TNF- $\alpha$, macrophage inflammatory protein (MIP)1, MIP2, and matrix metalloproteinase (MMP). Increased IL-6 secretion is linked to the abnormal long activation of the STAT3 signaling pathway. Prolonged STAT3 activation enhances the sustained expression of IL-6-induced proteins that play important roles in inflammation and carcinogenesis. ${ }^{68}$ As an inducer of INF- $\gamma$, IL-18 upregulates the expression of INF- $\gamma$ and subsequently activates several intrinsic cellular pathways such as Janus kinase (JAK)-STAT signaling. ${ }^{69}$ Activation of JAK-STAT signaling leads to nuclear translocation of the STAT1 protein, and increases the transcription regulation of INF- $\gamma$-induced genes, which are responsible for proliferation, differentiation, and cell death. Therefore, IL-18-INF- - -STAT1 axis activation is involved in 
colon carcinogenesis. ${ }^{67}$ Increasing the IL-18 secretion and STAT3 activation could positively regulate the expression of antimicrobial peptides Reg3 $\beta$ and Reg3 $\gamma$. The overexpression of Reg3 $\beta$ and Reg3 $\gamma$ will maintain the activation of STAT3 and AKT signaling in a feed-back loop, leading to the dysregulated crypt formation and increased susceptibility of CAC. ${ }^{70}$ Unlike IL-18, inflammasome-mediated IL- $1 \beta$ is not relevant to CAC, but was shown in sunburn-like inflammation and skin carcinogenesis. ${ }^{71}$ The activation of the inflammasome results in the enhanced expression of pro-inflammatory cytokines, especially IL-1 $\beta$, in mouse skin co-exposed to arsenic and UVB irradiation, followed by severe epidermal hyperplasia and DNA damage. ${ }^{72}$ Consequently, the suppression of the IL-1 $\beta$ signaling pathway can protect the keratinocyte from carcinogenesis. IL-1 $\beta$ may also play a role in angiogenesis and invasiveness. In melanoma, the activation of the NLRP3 inflammasome by opening P2X7/PANX1 results in the increased release of pro-inflammatory mediators, especially IL-1 $\beta$. The increased secretion of IL-1 $\beta$ correlates with the enhanced migration and metastatic potential of melanoma. ${ }^{73}$ Mouse lung cancer cells that constitutively express IL-1 $\beta$ are more aggressive and show an increased angiogenesis than those of non-transgenic counterpart control mice. ${ }^{74}$ Systemic inhibition of IL-1 $\beta$ with anakinra suppressed the growth and density of new vessels in IL-1 $\beta$-producing human tumor cell lines. ${ }^{75}$

Evidence for the pro-cancer roles of other NLRs is also published. NAIPs are a family of NLRs with seven paralogs in mice and only one in human (hNAIP). The involvement of NAIP in cancer progression has been investigated. The Naip allele is frequently methylated in normal oral mucosa tissues, however, NAIP expression was found to significantly increase in malignantly transformed oral squamous cell carcinomas compared with the corresponding oral malignant disorders. ${ }^{76}$
Another group indicated an elevated NAIP expression in response to androgen depletion in prostate cancer and a correlation with an increased NF- $\kappa B$ DNA-binding activity. ${ }^{77}$ The NAIP expression might be an important event in carcinogenesis. NLRP1 was the first member of the NLR family to be identified with the inflammasome assembly and CASP1 activation. ${ }^{13}$ Like NLRP3, NLRP6, and NLRC4, NLRP1 also displayed a protective role in an AOM/DSS chemical-induced colitis carcinogenesis model. The $N L R P 1$ allele was found more frequently in malignant mesothelioma ${ }^{78}$ and is associated with giant cell arteritis. ${ }^{79}$ This evidence for a pro-cancer role of NLRP1 in tumor progression is still poor, therefore, more experiments need to be performed. Beside NLR inflammasomes, the AIM2 inflammasome is also known as a suppressor in certain cancers. However, a recent study showed that AIM2 activation could enhance INF- $\gamma$-independently and -dependently stimulated genes, which are involved in carcinogenesis. This group also reported that AIM2 in inflammasome-mediated cell death was not found in colorectal cancer cells. ${ }^{80}$ Overall, the results discussed above indicate that inflammasome components and their products play crucial roles in both promoting and suppressing inflammation-associated carcinogenesis in stimuli, cancer and context-dependent manner.

\section{THERAPEUTIC APPROACHES TARGETING INFLAMMASOME}

Therapeutic approaches targeting the inflammasome have been developed to overcome human cancer (Table 2). Although inflammasomes have been revealed in diverse pathologies, common approaches can reduce and dampen the inflammation where appropriate. Several developed reagents that particularly target the inflammasome or CASP1 activity are limited by

Table 2. Potential therapeutic agents for targeting the inflammasome in carcinogenesis

\begin{tabular}{|c|c|c|c|}
\hline Agent & Target & Outcome and acting mechanism & Reference no. \\
\hline \multirow[t]{3}{*}{ Anakinra } & IL-1 $\beta$ receptor & $\begin{array}{l}\text { Inhibits the IL- } 6 \text { production and enhances the progress-free survival of patients with indolent } \\
\text { myeloma }\end{array}$ & 90 \\
\hline & & $\begin{array}{l}\text { Is considered as an attractive therapeutic approach by blocking the IL-1 } \beta \text {-mediated } \\
\text { IL- } 6 \text { production in Castleman's disease }\end{array}$ & 91 \\
\hline & & $\begin{array}{l}\text { Significantly suppresses the growth of breast cancer and bone metastasis through } \\
\text { reducing cell proliferation and angiogenesis }\end{array}$ & 92 \\
\hline Thalidomide & NLRP3 & Exerts inhibitory effects on the NLRP3 inflammasome through blocking of the CASP 1 activity & 82,83 \\
\hline CRID3 & NLRP3, AIM2 & Blocks the oligomerization of ASC to inhibit the inflammasome activation & 86,87 \\
\hline MCC950 & NLRP3 & $\begin{array}{l}\text { Is a small molecule inhibitor for the canonical and non-canonical NLRP3 activation to } \\
\text { block the oligomerization of ASC }\end{array}$ & 88 \\
\hline
\end{tabular}

CRID3, cytokine release inhibitory drug 3; MCC950, 1-(1,2,3,5,6,7-hexahydro-s-indacen-4-yl)-3-[4-(2-hydroxypropan-2-yl)furan-2-yl]sulfonylurea; IL, interleukin; NLRP, nucleotide-binding domain and leucine-rich repeat containing receptor protein; AIM, absent in melanoma; CASP1, caspase 1; ASC, apoptosis-associated speck-like protein containing a caspase recruitment and activation domain. 
off-target effects on different inflammasome components and caspases, as well as side effects from excessive inhibition of inflammasome activities. ${ }^{81}$ Thalidomide possesses the antiangiogenic activities to block myeloma growth in 25\% to $35 \%$ of patients with relapsed cancers. ${ }^{82}$ Although the anti-tumorigenic mechanism of thalidomide is not fully understood yet, thalidomide may function as immunomodulator through inhibition of NLRP3 inflammasome and IL- $1 \beta$ secretion. ${ }^{83}$ These anti-neoplastic effects of thalidomide suggest that this agent might act in myeloma through CASP1 inhibition and disruption of the IL-1 $\beta$-IL-6 loop. Because the P2RX7 receptor is involved in the NLRP3/CASP1 cascade for the immunogenicity of dead cancer cells, it is a potent target for chemotherapy. In mouse models, the inhibition of P2RX7 with oxidized ATP or VEGF-blocking antibody, Avastin, suppressed the tumor growth. ${ }^{84}$ In contrast, inactivation of the P2RX7 receptor inhibits inflammation, but promotes tumor incidence in colitis-associated carcinogenesis model. ${ }^{85}$ Therefore, more in-depth studies are required to use the P2RX7 inhibitor for the treatment of human cancers. As a trial for developing anti-inflammatory drugs, researchers designed the cytokine release inhibitory drug 3 (CRID3; CP-456,773), which blocks ASC oligomerization at the initial step of inflammasome activation and modulates the progression of the disease. ${ }^{86,87}$ Due to the involvement of oligomerization of ASC in inflammasome activation, blocking this process by CRID3 may provide another option to treat human cancer. To modulate the NLRP3 inflammasome-involved human diseases, a selective small-molecule inhibitor of NLRP3 (MCC950) was also developed and validated in several disease models. ${ }^{88}$ Because this molecule is specific for NLRP3 inflammasome rather than other inflammasomes, it can be used as potent therapeutics against NLRP3 inflammasome-involved human cancer.

Many groups have paid attention to the development of compounds to inhibit the IL-1 $\beta$ signaling activity as another strategy for inflammasome inhibition as cancer therapeutics. The specific inhibitors of IL-1 $\beta$ signaling include monoclonal antibodies and recombinant derivatives of IL-1RN, which neutralize both IL- $1 \alpha$ and IL-1 $\beta$, respectively. ${ }^{89}$ Treatment of the IL-1 $\beta$ antagonist has shown an effectiveness in a variety of disorders, which results in a significant benefit for patients. Because the myeloma-derived IL-1 $\beta$ induced the IL- 6 production in bone marrow stroma cells, blocking the IL-1 $\beta$ signaling by the IL-1 $\beta$ receptor antagonist, anakinra, showed an inhibitory efficacy to the IL- 6 production and enhanced the progress-free survival of patients with indolent myeloma in clinical treatment. ${ }^{90}$ Treatment with anakinra has also been reported to be an attractive therapeutic approach for refractory multicentric Castleman's disease, a very rare lymphoproliferative disorder. ${ }^{91}$ This anti-tumorigenic activity of anakinra may originate from blocking of the IL-1 $\beta$-mediated production of IL- 6 , which is a key factor for inflammation-associated cancer. Anakinra also significantly reduced the growth of breast cancer and bone metastasis. It did not promote the apoptosis of tumor cells, but markedly reduced the proliferation and angiogenesis in addition to exerting significant effects on the tumor environment by reducing bone turnover effectors, such as IL- $1 \beta$ and TNF- $\alpha{ }^{92}$

While chemotherapy treatments have been successfully performed against many types of cancer, they often cause severe side effects which depend on the cancer types, locations, kinds of drug, and doses. Five-floururacil (5-FU), doxorubicin, and bleomycin are the most common drugs for cancer chemotherapy that positively regulate NLRP3 inflammasome activities. Co-treatment of the IL-1 $\beta$ inhibitors might be useful for modulating genotoxic chemotherapy-induced side effects. Pretreatment of IL-1 $\beta$ inhibitor (IL-1Ra) reduced the acute lethal toxicity and bone marrow suppression, induced by 5-FU in hematopoietic cells, further supporting this idea. ${ }^{93}$ IL-1 $\beta$ inhibitors can also reduce doxorubicin-triggered cardiotoxicity and dose-dependent congestive heart failure. ${ }^{94}$ Pulmonary fibrosis elicited by bleomycin can be eliminated via cotreatment of IL- $1 \beta$ inhibitor. ${ }^{95}$ In contrast, IL-1 $\beta$ inhibitors suppress the anti-cancer effects of oxaliplatin or anthracyclines. ${ }^{96}$ Therefore, clinical use of IL-1 $\beta$ inhibitors should be carefully performed by considering the context and type of cancer.

\section{CONCLUSIONS}

As described above, the inflammasome signaling is closely associated with many human cancers. The activation of inflammasome with various insults enhances the secretion of inflammatory cytokines, leading to infiltration of more immune cells and resulting in the generation and maintenance of an inflammatory microenvironment surrounding cancer cells. In the process of carcinogenesis, the inflammasome also inhibits the anti-carcinogenic activity of NK cell- and $\mathrm{T}$ cell-mediated immunosurveillance. It also promotes the angiogenesis and metastasis of cancer through the IL-1 $\beta$-dependent upregulation of CCL2, COX 2, and fibroblast growth factor 2. In contrast to its pro-carcinogenic activity, the inflammasome also has the ability to suppress the cancer growth in many studies. Its anti-carcinogenic activity has its origin from the pyroptotic cell death of premalignant cells and the suppression of immune responses 
against potentially pathogenic microbes through the recruitment of cytotoxic immune cells. The inflammasome is also involved in chemotherapy-induced anticancer immune responses by sensing immunogenic signals that are exposed to or released from dying cells.

Although many achievements were accomplished during the research of inflammasomes and carcinogenesis, there are still many questions to be answered. Why does inflammasome have an ambivalent activity in cancer development? It is well known that each protein has a specific function depending on existing tissues. ASC functions as an oncoprotein in myeloid cells for skin cancer but inhibits skin carcinogenesis in keratinocyte. The NLRP3 inflammasome functions mainly in myeloid cells while the NLRC4 inflammasome works in epithelial cells in colon carcinogenesis. Occasionally the same proteins such as TGF- $\beta$ show opposite activities in the process of carcinogenesis depending on cancer stage. Further studies are required to confirm whether the same inflammasome shows different activities on cancer, depending on the grade. Are all 22 NLRs involved in the modulation of the inflammatory signaling for human carcinogenesis? The regulatory mechanism between different NLR members is still unclear. Only several NLRs have been studied regarding ligands, signaling pathways, and role in human diseases. If we can clarify the detailed regulatory mechanism for all NLRs, we will better understand the more delicate functions of the inflammasome in carcinogenesis. What is the molecular mechanism of the activation or suppression of inflammasomes in the cancer microenvironment? If we identify the regulatory factors for the inflammasome, those factors can be used as biomarkers for the prognosis of and therapeutic targets against various cancers. Although there are still many knowledge gaps for the function of the inflammasome in carcinogenesis, understanding its signaling pathway, regulatory mechanism, and pathological significance will give us a chance to develop new therapeutic strategies for the prevention and treatment of human malignant cancers.

\section{ACKNOWLEDGMENTS}

This work was supported by the National Research Foundation of Korea (NRF) grant funded by the Korea government (2016R1A2B2008007) to S Hong.

\section{CONFLICTS OF INTEREST}

No potential conflicts of interest were disclosed.

\section{REFERENCES}

1. Odegaard JI, Chawla A. The immune system as a sensor of the metabolic state. Immunity 2013;38:644-54.

2. Hanahan D, Weinberg RA. Hallmarks of cancer: the next generation. Cell 2011;144:646-74.

3. Kim S, Takahashi H, Lin WW, Descargues P, Grivennikov S, Kim Y, et al. Carcinoma-produced factors activate myeloid cells through TLR2 to stimulate metastasis. Nature 2009:457:102-6.

4. Mantovani A, Allavena P, Sica A, Balkwill F. Cancer-related inflammation. Nature 2008:454:436-44.

5. Colotta F, Allavena P, Sica A, Garlanda C, Mantovani A. Cancer-related inflammation, the seventh hallmark of cancer: links to genetic instability. Carcinogenesis 2009;30:1073-81.

6. Oguma K, Oshima H, Aoki M, Uchio R, Naka K, Nakamura S, et al. Activated macrophages promote Wnt signalling through tumour necrosis factor-alpha in gastric tumour cells. EMBO J 2008; 27:1671-81.

7. Roberts $A B$, Wakefield LM. The two faces of transforming growth factor beta in carcinogenesis. Proc Natl Acad Sci U S A 2003; 100:8621-3.

8. Zlotnik A, Burkhardt AM, Homey B. Homeostatic chemokine receptors and organ-specific metastasis. Nat Rev Immunol 2011:11:597-606.

9. Mukherjee D, Zhao J. The role of chemokine receptor CXCR4 in breast cancer metastasis. Am J Cancer Res 2013;3:46-57.

10. Zipin-Roitman A, Meshel T, Sagi-Assif O, Shalmon B, Avivi C, Pfeffer RM, et al. CXCL10 promotes invasion-related properties in human colorectal carcinoma cells. Cancer Res 2007;67: 3396-405.

11. Kanneganti TD, Lamkanfi M, Núñez G. Intracellular NOD-like receptors in host defense and disease. Immunity 2007;27:549-59.

12. Takeda $\mathrm{K}$, Akira $\mathrm{S}$. Toll-like receptors in innate immunity. Int Immunol 2005;17:1-14.

13. Martinon F, Burns K, Tschopp J. The inflammasome: a molecular platform triggering activation of inflammatory caspases and processing of proIL-beta. Mol Cell 2002;10:417-26.

14. Ting JP, Davis BK. CATERPILLER: a novel gene family important in immunity, cell death, and diseases. Annu Rev Immunol 2005:23:387-414.

15. Franchi L, Eigenbrod T, Muñoz-Planillo R, Nuñez G. The inflammasome: a caspase-1-activation platform that regulates immune responses and disease pathogenesis. Nat Immunol 2009;10:241-7.

16. Zitvogel L, Kepp O, Galluzzi L, Kroemer G. Inflammasomes in carcinogenesis and anticancer immune responses. Nat Immunol 2012;13:343-51.

17. Gringhuis SI, Kaptein TM, Wevers BA, Theelen B, van der Vlist M, Boekhout T, et al. Dectin-1 is an extracellular pathogen sensor for the induction and processing of IL-1 $\beta$ via a noncanonical caspase-8 inflammasome. Nat Immunol 2012;13:246-54.

18. Sagulenko V, Thygesen SJ, Sester DP, Idris A, Cridland JA, Vajjhala PR, et al. AIM2 and NLRP3 inflammasomes activate both apoptotic and pyroptotic death pathways via ASC. Cell Death Differ 2013;20:1149-60.

19. Broz P, Monack DM. Molecular mechanisms of inflammasome activation during microbial infections. Immunol Rev 2011;243:174-90. 
20. Kayagaki N, Warming S, Lamkanfi M, Vande Walle L, Louie S, Dong J, et al. Non-canonical inflammasome activation targets caspase-11. Nature 2011;479:117-21.

21. Sharma N, Jha S. NLR-regulated pathways in cancer: opportunities and obstacles for therapeutic interventions. Cell Mol Life Sci 2016;73:1741-64.

22. Zhiyu W, Wang N, Wang Q, Peng C, Zhang J, Liu P, et al. The inflammasome: an emerging therapeutic oncotarget for cancer prevention. Oncotarget 2016;7:50766-80.

23. Chavarría-Smith J, Vance RE. Direct proteolytic cleavage of NLRP1B is necessary and sufficient for inflammasome activation by anthrax lethal factor. PLoS Pathog 2013;9:e1003452.

24. Hsu LC, Ali SR, McGillivray S, Tseng PH, Mariathasan S, Humke EW, et al. A NOD2-NALP1 complex mediates caspase-1-dependent IL-1beta secretion in response to Bacillus anthracis infection and muramyl dipeptide. Proc Natl Acad Sci U S A 2008;105:7803-8.

25. Kovarova M, Hesker PR, Jania L, Nguyen M, Snouwaert JN, Xiang $Z$, et al. NLRP1-dependent pyroptosis leads to acute lung injury and morbidity in mice. J Immunol 2012;189:2006-16.

26. Tan CC, Zhang JG, Tan MS, Chen H, Meng DW, Jiang T, et al. NLRP1 inflammasome is activated in patients with medial temporal lobe epilepsy and contributes to neuronal pyroptosis in amygdala kindling-induced rat model. J Neuroinflammation 2015;12:18.

27. Kanneganti TD, Ozören N, Body-Malapel M, Amer A, Park JH, Franchi L, et al. Bacterial RNA and small antiviral compounds activate caspase-1 through cryopyrin/Nalp3. Nature 2006;440:233-6.

28. Mariathasan S, Weiss DS, Newton K, McBride J, O'Rourke K, Roose-Girma M, et al. Cryopyrin activates the inflammasome in response to toxins and ATP. Nature 2006:440:228-32.

29. Muruve DA, Pétrilli V, Zaiss AK, White LR, Clark SA, Ross PJ, et al. The inflammasome recognizes cytosolic microbial and host DNA and triggers an innate immune response. Nature 2008:452:103-7.

30. Martinon F, Pétrilli V, Mayor A, Tardivel A, Tschopp J. Gout-associated uric acid crystals activate the NALP3 inflammasome. Nature 2006;440:237-41.

31. Lee GS, Subramanian N, Kim AI, Aksentijevich I, Goldbach-Mansky R, Sacks DB, et al. The calcium-sensing receptor regulates the NLRP3 inflammasome through Ca2 + and CAMP. Nature 2012;492:123-7.

32. Zhou R, Yazdi AS, Menu P, Tschopp J. A role for mitochondria in NLRP3 inflammasome activation. Nature 2011;469:221-5.

33. Juliana C, Fernandes-Alnemri T, Kang S, Farias A, Qin F, Alnemri ES. Non-transcriptional priming and deubiquitination regulate NLRP3 inflammasome activation. J Biol Chem 2012;287:36617-22.

34. Hao H, Cao L, Jiang C, Che Y, Zhang S, Takahashi S, et al. Farnesoid X receptor regulation of the NLRP3 inflammasome underlies cholestasis-associated sepsis. Cell Metab 2017;25:856-67.e5.

35. Franchi L, Kamada N, Nakamura Y, Burberry A, Kuffa P, Suzuki S, et al. NLRC4-driven production of IL-1 $\beta$ discriminates between pathogenic and commensal bacteria and promotes host intestinal defense. Nat Immunol 2012;13:449-56.

36. Zhao Y, Yang J, Shi J, Gong YN, Lu Q, Xu H, et al. The NLRC4 inflammasome receptors for bacterial flagellin and type III secretion apparatus. Nature 2011:477:596-600.

37. Rauch I, Deets KA, Ji DX, von Moltke J, Tenthorey JL, Lee AY, et al. NAIP-NLRC4 inflammasomes coordinate intestinal epithelial cell expulsion with eicosanoid and IL-18 release via activation of caspase-1 and -8. Immunity 2017;46:649-59.
38. Wlodarska M, Thaiss CA, Nowarski R, Henao-Mejia J, Zhang JP, Brown EM, et al. NLRP6 inflammasome orchestrates the colonic host-microbial interface by regulating goblet cell mucus secretion. Cell 2014;156:1045-59.

39. Bürckstümmer T, Baumann C, Blüml S, Dixit E, Dürnberger G, Jahn $\mathrm{H}$, et al. An orthogonal proteomic-genomic screen identifies AIM2 as a cytoplasmic DNA sensor for the inflammasome. Nat Immunol 2009;10:266-72.

40. Fernandes-Alnemri T, Yu JW, Datta P, Wu J, Alnemri ES. AIM2 activates the inflammasome and cell death in response to cytoplasmic DNA. Nature 2009;458:509-13.

41. Hanamsagar R, Aldrich A, Kielian T. Critical role for the AIM2 inflammasome during acute CNS bacterial infection. J Neurochem 2014:129:704-11.

42. Strasser A, Cory S, Adams JM. Deciphering the rules of programmed cell death to improve therapy of cancer and other diseases. EMBO J 2011;30:3667-83.

43. Vandenabeele P, Galluzzi L, Vanden Berghe T, Kroemer G. Molecular mechanisms of necroptosis: an ordered cellular explosion. Nat Rev Mol Cell Biol 2010;11:700-14.

44. Bergsbaken T, Fink SL, Cookson BT. Pyroptosis: host cell death and inflammation. Nat Rev Microbiol 2009;7:99-109.

45. Winter RN, Kramer A, Borkowski A, Kyprianou N. Loss of caspase- 1 and caspase- 3 protein expression in human prostate cancer. Cancer Res 2001;61:1227-32.

46. Winter RN, Rhee JG, Kyprianou N. Caspase-1 enhances the apoptotic response of prostate cancer cells to ionizing radiation. Anticancer Res 2004:24:1377-86.

47. Feng Q, Li P, Salamanca C, Huntsman D, Leung PC, Auersperg N. Caspase-1alpha is down-regulated in human ovarian cancer cells and the overexpression of caspase-1alpha induces apoptosis. Cancer Res 2005:65:8591-6.

48. Hu B, Elinav E, Huber S, Booth CJ, Strowig T, Jin C, et al. Inflammation-induced tumorigenesis in the colon is regulated by caspase-1 and NLRC4. Proc Natl Acad Sci U S A 2010;107:21635-40.

49. Faustin B, Reed JC. Sunburned skin activates inflammasomes. Trends Cell Biol 2008;18:4-8.

50. Sollberger G, Strittmatter GE, Grossi S, Garstkiewicz M, Auf dem Keller U, French LE, et al. Caspase-1 activity is required for UVB-induced apoptosis of human keratinocytes. J Invest Dermatol 2015;135:1395-404.

51. Zhong Y, Kinio A, Saleh M. Functions of NOD-like receptors in human diseases. Front Immunol 2013;4:333.

52. Allen IC, TeKippe EM, Woodford RM, Uronis JM, Holl EK, Rogers $\mathrm{AB}$, et al. The NLRP3 inflammasome functions as a negative regulator of tumorigenesis during colitis-associated cancer. J Exp Med 2010;207:1045-56

53. Zaki MH, Boyd KL, Vogel P, Kastan MB, Lamkanfi M, Kanneganti TD. The NLRP3 inflammasome protects against loss of epithelial integrity and mortality during experimental colitis. Immunity 2010;32:379-91.

54. Dupaul-Chicoine J, Arabzadeh A, Dagenais M, Douglas T, Champagne C, Morizot A, et al. The Nlrp3 inflammasome suppresses colorectal cancer metastatic growth in the liver by promoting natural killer cell tumoricidal activity. Immunity 2015:43:751-63.

55. Huber S, Gagliani N, Zenewicz LA, Huber FJ, Bosurgi L, Hu B, et al. IL-22BP is regulated by the inflammasome and modulates tumorigenesis in the intestine. Nature 2012;491:259-63. 
56. Ghiringhelli F, Apetoh L, Tesniere A, Aymeric L, Ma Y, Ortiz C, et al. Activation of the NLRP3 inflammasome in dendritic cells induces IL-1beta-dependent adaptive immunity against tumors. Nat Med 2009;15:1170-8.

57. Chow MT, Sceneay J, Paget C, Wong CS, Duret H, Tschopp J, et al. NLRP3 suppresses NK cell-mediated responses to carcinogen-induced tumors and metastases. Cancer Res 2012;72:5721-32.

58. Wei Q, Mu K, Li T, Zhang Y, Yang Z, Jia X, et al. Deregulation of the NLRP3 inflammasome in hepatic parenchymal cells during liver cancer progression. Lab Invest 2014;94:52-62.

59. Hu B, Elinav E, Flavell RA. Inflammasome-mediated suppression of inflammation-induced colorectal cancer progression is mediated by direct regulation of epithelial cell proliferation. Cell Cycle 2011;10:1936-9.

60. Chen GY, Liu M, Wang F, Bertin J, Núñez G. A functional role for Nlrp6 in intestinal inflammation and tumorigenesis. J Immunol 2011;186:7187-94

61. Normand S, Delanoye-Crespin A, Bressenot A, Huot L, Grandjean T, Peyrin-Biroulet L, et al. Nod-like receptor pyrin domain-containing protein 6 (NLRP6) controls epithelial self-renewal and colorectal carcinogenesis upon injury. Proc Natl Acad Sci U S A 2011;108:9601-6.

62. Zaki MH, Vogel P, Malireddi RK, Body-Malapel M, Anand PK, Bertin J, et al. The NOD-like receptor NLRP12 attenuates colon inflammation and tumorigenesis. Cancer Cell 2011;20:649-60.

63. Chen L, Wilson JE, Koenigsknecht MJ, Chou WC, Montgomery SA, Truax AD, et al. NLRP12 attenuates colon inflammation by maintaining colonic microbial diversity and promoting protective commensal bacterial growth. Nat Immunol 2017;18:541-51.

64. Cook GP, Savic S, Wittmann M, McDermott MF. The NLRP3 inflammasome, a target for therapy in diverse disease states. Eur J Immunol 2010;40:631-4.

65. van Deventer HW, Burgents JE, Wu QP, Woodford RM, Brickey WJ, Allen IC, et al. The inflammasome component NLRP3 impairs antitumor vaccine by enhancing the accumulation of tumor-associated myeloid-derived suppressor cells. Cancer Res 2010; 70:10161-9.

66. Chow MT, Tschopp J, Möller A, Smyth MJ. NLRP3 promotes inflammation-induced skin cancer but is dispensable for asbestos-induced mesothelioma. Immunol Cell Biol 2012;90:983-6.

67. Zaki MH, Lamkanfi M, Kanneganti TD. The Nlrp3 inflammasome: contributions to intestinal homeostasis. Trends Immunol 2011; 32:171-9.

68. Wang Y, van Boxel-Dezaire AH, Cheon H, Yang J, Stark GR. STAT3 activation in response to IL- 6 is prolonged by the binding of IL- 6 receptor to EGF receptor. Proc Natl Acad Sci U S A 2013;110: $16975-80$

69. Zaki MH, Lamkanfi M, Kanneganti TD. Inflammasomes and intestinal tumorigenesis. Drug Discov Today Dis Mech 2011:8:e71-8.

70. Ratsimandresy RA, Indramohan M, Dorfleutner A, Stehlik C. The AIM2 inflammasome is a central regulator of intestinal homeostasis through the IL-18/IL-22/STAT3 pathway. Cell Mol Immunol 2017; 14:127-42.

71. Feldmeyer L, Keller M, Niklaus G, Hohl D, Werner S, Beer HD. The inflammasome mediates UVB-induced activation and secretion of interleukin-1beta by keratinocytes. Curr Biol 2007;17:1140-5.

72. Lin C, Zhang J. Inflammasomes in inflammation-induced cancer. Front Immunol 2017;8:271.

73. Schneider SL, Ross AL, Grichnik JM. Do inflammatory pathways drive melanomagenesis? Exp Dermatol 2015;24:86-90.

74. Nakao S, Kuwano T, Tsutsumi-Miyahara C, Ueda S, Kimura YN, Hamano $\mathrm{S}$, et al. Infiltration of COX-2-expressing macrophages is a prerequisite for IL-1 beta-induced neovascularization and tumor growth. J Clin Invest 2005;115:2979-91.

75. Weinreich DM, Elaraj DM, Puhlmann M, Hewitt SM, Carroll NM, Feldman ED, et al. Effect of interleukin 1 receptor antagonist gene transduction on human melanoma xenografts in nude mice. Cancer Res 2003;63:5957-61.

76. Hebb AL, Moore CS, Bhan V, Campbell T, Fisk JD, Robertson HA, et al. Expression of the inhibitor of apoptosis protein family in multiple sclerosis reveals a potential immunomodulatory role during autoimmune mediated demyelination. Mult Scler 2008; 14:577-94

77. Chiu HH, Yong TM, Wang J, Wang Y, Vessella RL, Ueda T, et al. Induction of neuronal apoptosis inhibitory protein expression in response to androgen deprivation in prostate cancer. Cancer Lett 2010;292:176-85.

78. Girardelli M, Maestri I, Rinaldi RR, Tognon M, Boldorini R, Bovenzi M, et al. NLRP1 polymorphisms in patients with asbestos-associated mesothelioma. Infect Agent Cancer 2012;7:25.

79. Serrano A, Carmona FD, Castañeda S, Solans R, Hernández-Rodríguez J, Cid MC, et al. Evidence of association of the NLRP1 gene with giant cell arteritis. Ann Rheum Dis 2013; 72:628-30.

80. Lee J, Li L, Gretz N, Gebert J, Dihlmann S. Absent in Melanoma 2 (AIM2) is an important mediator of interferon-dependent and -independent HLA-DRA and HLA-DRB gene expression in colorectal cancers. Oncogene 2012;31:1242-53.

81. Green DR, Kroemer G. Pharmacological manipulation of cell death: clinical applications in sight? J Clin Invest 2005;115:2610-7.

82. Palumbo A, Facon T, Sonneveld P, Bladè J, Offidani M, Gay F, et al. Thalidomide for treatment of multiple myeloma: 10 years later. Blood 2008;111:3968-77.

83. Keller M, Sollberger G, Beer HD. Thalidomide inhibits activation of caspase-1. J Immunol 2009;183:5593-9.

84. Adinolfi E, Raffaghello L, Giuliani AL, Cavazzini L, Capece M, Chiozzi P, et al. Expression of P2X7 receptor increases in vivo tumor growth. Cancer Res 2012;72:2957-69.

85. Hofman P, Cherfils-Vicini J, Bazin M, Ilie M, Juhel T, Hébuterne X, et al. Genetic and pharmacological inactivation of the purinergic P2RX7 receptor dampens inflammation but increases tumor incidence in a mouse model of colitis-associated cancer. Cancer Res 2015;75:835-45.

86. López-Castejón G, Pelegrín P. Current status of inflammasome blockers as anti-inflammatory drugs. Expert Opin Investig Drugs 2012;21:995-1007.

87. Ludwig-Portugall I, Bartok E, Dhana E, Evers BD, Primiano MJ, Hall JP, et al. An NLRP3-specific inflammasome inhibitor attenuates crystal-induced kidney fibrosis in mice. Kidney Int 2016;90:525-39

88. Coll RC, Robertson AA, Chae JJ, Higgins SC, Muñoz-Planillo R, Inserra MC, et al. A small-molecule inhibitor of the NLRP3 inflammasome for the treatment of inflammatory diseases. Nat Med 2015;21:248-55.

89. Dinarello CA. Interleukin-1 in the pathogenesis and treatment of inflammatory diseases. Blood 2011;117:3720-32.

90. Lust JA, Lacy MQ, Zeldenrust SR, Dispenzieri A, Gertz MA, Witzig $\mathrm{TE}$, et al. Induction of a chronic disease state in patients with 
smoldering or indolent multiple myeloma by targeting interleukin 1 \{beta\}-induced interleukin 6 production and the myeloma proliferative component. Mayo Clin Proc 2009;84:114-22.

91. El-Osta H, Janku F, Kurzrock R. Successful treatment of Castleman's disease with interleukin-1 receptor antagonist (Anakinra). Mol Cancer Ther 2010;9:1485-8.

92. Holen I, Lefley DV, Francis SE, Rennicks S, Bradbury S, Coleman $\mathrm{RE}$, et al. IL-1 drives breast cancer growth and bone metastasis in vivo. Oncotarget 2016;7:75571-84.

93. Zhang J, Xiang D, Zhu S, Mao W, Lu H, Wu M, et al. Interleukin 1 receptor antagonist inhibits normal hematopoiesis and reduces lethality and bone marrow toxicity of 5-fluouracil in mouse.
Biomed Pharmacother 2009;63:501-8

94. Zhu J, Zhang J, Zhang L, Du R, Xiang D, Wu M, et al. Interleukin-1 signaling mediates acute doxorubicin-induced cardiotoxicity. Biomed Pharmacother 2011;65:481-5.

95. Gasse P, Mary C, Guenon I, Noulin N, Charron S, Schnyder-Candrian S, et al. IL-1R1/MyD88 signaling and the inflammasome are essential in pulmonary inflammation and fibrosis in mice. J Clin Invest 2007;117:3786-99.

96. Mattarollo SR, Loi S, Duret H, Ma Y, Zitvogel L, Smyth MJ. Pivotal role of innate and adaptive immunity in anthracycline chemotherapy of established tumors. Cancer Res 2011;71:4809-20. 